\title{
Wrinkled petals and stamens 1, is required for the morphogenesis of petals and stamens in Lotus japonicus
}

\author{
Jiang Hua Chen ${ }^{1}$, Ji Liang Pang ${ }^{2}$, Li Lin Wang ${ }^{2}$, Yong Hai Luo ${ }^{1}, \mathrm{Xin}_{\mathrm{Li}}^{3}$, Xiang Ling Cao ${ }^{1}$, Kui Lin ${ }^{3}$, Wei Ma \\ Xiao $\mathrm{He} \mathrm{Hu}^{1}$, Da Luo ${ }^{1,3}$ \\ ${ }^{I}$ National Laboratory of Plant Molecular Genetics, Institute of Plant Physiology and Ecology, Shanghai Institutes for Biological Sci- \\ ences, and Graduate School of the Chinese Academy of Sciences, Chinese Academy of Sciences, 300 Fenglin Road, Shanghai 200032, \\ China; ${ }^{2}$ School of Life Science, Hangzhou Normal College, 16 Xuelin Road, Hangzhou 310018, China; ${ }^{3}$ School of Life Science and \\ Biotechnology, Shanghai Jiao Tong University, 1954 Huashan Road, Shanghai 200030, China
}

Although much progress has been made in understanding how floral organ identity is determined during the floral development, less is known about how floral organ is elaborated in the late floral developmental stages. Here we describe a novel floral mutant, wrinkled petals and stamens1 (wps 1), which shows defects in the development of petals and stamens. Genetic analysis indicates that wps 1 mutant is corresponding to a single recessive locus at the long arm of chromosome 3. The early development of floral organs in wps 1 mutant is similar to that in wild type, and the malfunction of the mutant commences in late developmental stages, displaying a defect on the appearance of petals and stamens. In the mature flower, petals and stamen filaments in the mutant are wrinkled or folded, and the cellular morphology under L1 layer of petals and stamen filaments is abnormal. It is found that the expression patterns of floral organ identity genes are not affected in wps 1 mutants compared with that of wild type, consistent with the unaltered development of all floral organs. Furthermore, the identities of epidermal cells in different type of petals are maintained. The histological analysis shows that in wps 1 flowers all petals are irregularly folded, and there are knotted structures in the petals, while the shape and arrangement of inner cells are malformed and unorganized. Based on these results, we propose that Wps 1 acts downstream to the class B floral organ identity genes, and functions to modulate the cellular differentiation during the late flower developmental stages.

Cell Research (2006) 16:499-506. doi:10.1038/sj.cr.7310061; published online 15 May 2006

Keywords: Lotus japonicus, wps 1 mutant, $\mathrm{ABC}$ model, floral organ differentiation

\section{Introduction}

In the past twenty years, the flower development study has been made great progress in two model systems, Arabidopsis thaliana and Antirrhinum majus. The classical 'ABC model' has been widely tested and proven to be generally applicable in controlling floral organ identity during floral development among angiosperms $[1,2]$. This

Correspondence: Da Luo

Tel: +86-21-5492-4108; Fax: +86-21-5492-4106;

E-mail: dluo@sibs.ac.cn

Received 18 Jan 2006; revised 1 Mar 2006; accepted 21 Mar 2006; published online 15 May 2006 model states that floral organ identity is specified by different combinations of the activities of the A, B and C class homeotic genes. In Arabidopsis, each of these functions operates in two adjacent whorls of flower. Furthermore, A and $\mathrm{C}$ functions antagonize each other. Thus, $\mathrm{A}$ function alone controls the identity of sepals, A and B determine the identity of petals, B plus C specify the identity of stamens, and $\mathrm{C}$ alone directs carpel identity. $\mathrm{D}$-function genes controlling the ovule identity and E-function genes that function as co-factors with $\mathrm{ABC}$ genes in the inner three whorls of flower, were introduced later [3,4]. So far, few direct targets of the ABC function genes are identified [5]. Functional analysis of targets of floral organ identity genes has been hampered by the lack of mutants; however, this 
situation has been changed significantly due to the application of a combination of forward genetic and whole-genome approaches. Some candidate target genes were identified by analysis of expression profiling $[6,7]$.
Lotus japonicus [8], a model plant in legumes, is an amenable system to study the nodulation and other development processes [9-11]. The whole-genome sequencing project has been carried out [12], and several L. japonicus

Table 1 The information of SSR markers used in wps 1 mapping work

\begin{tabular}{|c|c|c|c|c|c|c|}
\hline \multirow[t]{2}{*}{$\overline{\text { Chr. }}$} & \multirow[t]{2}{*}{$* \mathrm{P}(\mathrm{cM})$} & \multirow[t]{2}{*}{ Marker No. } & \multicolumn{2}{|c|}{ Product Size } & \multirow[t]{2}{*}{ Fw_primer (5' to $\left.3^{\prime}\right)$} & \multirow[t]{2}{*}{ Rv_primer (5' to 3') } \\
\hline & & & MG-20 & B-129 & & \\
\hline 1 & 0.0 & TM0123 & 182 & 160 & AATGAAAAGATGTATAACAGTCAC & TTTTCCAATCGAACTTGCCG \\
\hline 1 & 21.7 & TM0193 & 110 & 90 & GAGGATCTAAATCCATAGGG & AGTAATCTTAACCACCTATATGATG \\
\hline 1 & 45.6 & TM0117 & 127 & 155 & GGATATGCTCTGATACCATATTAG & TGGTCATACTGTGTAACTGAATAAG \\
\hline 2 & 4.0 & TM0134 & 135 & 155 & CCAGCTTGAGCAGTATAATGC & CCATAATGCATGAACTTTGTC \\
\hline 2 & 15.6 & TM0065 & 114 & 124 & AATGCTAGTTAAGCGCTCTC & CCCAAAGGTCTATAATTATAAGG \\
\hline 2 & 27.4 & TM0225 & 125 & 119 & GCAGCAACTTAAAAGTAAGC & CTATTGATTGGTTCATTCCG \\
\hline 2 & 36.3 & TM0124 & 139 & 143 & TCGAGTTCACGCAGTAAACC & ACAACAAACAGTGGATACCC \\
\hline 3 & 26.6 & TM0190 & 219 & 209 & TTGCATTAGTGGAAGTTTGG & CCTTTCGTGATTATGGAGAG \\
\hline 3 & 32.8 & TM0155 & 115 & 135 & GACACAACGTATAGCAGACA & GAATGGAGAATGATGATTGG \\
\hline 3 & 42.4 & TM0142 & 140 & 132 & CACATACACATTTGCCGAAG & CGCTTGCAGTGATTCTAACC \\
\hline 3 & 56.7 & TM0049 & 141 & 159 & TGGGTTAGCTTACCTGTTTC & ATGTCCTGATCAAATGTTTC \\
\hline 3 & 66.8 & TM0203 & 151 & 179 & CTGTTTTGACGAATCGATAG & TACGAAGGTTTCTTCATTCC \\
\hline 3 & 84.8 & TM0127 & 131 & 119 & CACATTGCGTTTGAAACTCG & GGTTCTGCTCAGCTTCGGTG \\
\hline 4 & 2.0 & TM0288 & 139 & 142 & GGGCATTTGCTGTTAGATTC & AACAAACCAAGCTCGATGAC \\
\hline 4 & 8.8 & TM0182 & 129 & 143 & TCTGAGCACAACCTACTACTG & TGTAATCAATAACATAGTCATTGC \\
\hline 4 & 24.2 & TM0247 & 133 & 155 & ATTGGAATAAATGAACATATATAGC & ATACCCCAACAAATTTTGAC \\
\hline 5 & 49.0 & TM0072 & 129 & 159 & TTATGGTGCTGTATGAGTATG & СTTATGAAACTTAAGCCCTG \\
\hline 5 & 56.6 & TM0146 & 147 & 159 & ATGTTACCTGATGGCGACGG & GTCCAATAATACCCTCCAAG \\
\hline 6 & 8.1 & TM0014 & 159 & 153 & AACCACAGACAAGATTATCG & TCATCATGATAATCATTCAAC \\
\hline 6 & 29.6 & TM0057 & 138 & 132 & CAAAGATAAATGCAGATGGC & СTTTCTATAAACAGTGAAACTGG \\
\hline 6 & 38.1 & TM0140 & 138 & 128 & GGAAATCAATTTCGGGAGGC & TGGACAGTAATAAATACATTCG \\
\hline 6 & 48.6 & TM0066 & 123 & 129 & CTTACCCGTGACTTAACTTG & CTAAAGGTTGCTTTCATGTC \\
\hline 6 & 59.6 & TM0336 & 164 & 180 & TGAGCCAATCAGAACCTATG & CATGTGCCTGAAATGTTACC \\
\hline 6 & 68.1 & TM0314 & 122 & 128 & TGTGATTAGTGATTAGAAAGTGAG & TTTGACCAAACTTCCTTCAC \\
\hline 3 & 80.8 & $* * \mathrm{TM} 0135$ & 122 & 116 & GTGGAAACCTTAACAATGTG & ACACAAGAAACCTAAACAAATG \\
\hline
\end{tabular}

*For the position information of each marker: http://www.kazusa.or.jp/lotus/

**TM0135: A maker located in the chromosome 3 for the fine mapping of wps 1 . 
mutant populations have been generated with many mutant classes being identified $[9,13,14]$. And two genetic linkage maps of L. japonicus have been made [15, 16], which have been facilitated the mapping and cloning projects of various important genes. The floral patterning in Papilionoideae plants is unique in terms of initiation timing, arrangement and floral organ number from other dicot model systems [17-19]. Our previous work has demonstrated that L. japonicus is an excellent system to dissect the components in the pathway to control floral development $[20,21]$.

A large scale of ethyl methanesulfonate (EMS) mutagenesis experiment has been conducted to screen mutations which affect floral development at various stages in L. japonicus. We report here a novel floral mutant in $L$. japonicus, wrinkled petals and stamens1 (wps1), which affects the development of petals and stamens in the late floral developmental stages, giving rise to folded petals and stamen filaments. Genetic analysis shows that the wps $1 \mathrm{mu}-$ tant is corresponding to a single recessive nuclear mutation. Further analysis indicates that the identities of petals and stamens are not changed; however, the cellular morphology of the inner layers in petals and stamens is abnormal. These data indicate that Wps1 is a potential downstream component of the B function, which could take part in the control of petal differentiation by regulating cellular differentiation in the late floral developmental stages.

\section{Materials and methods}

\section{Plant material and growth conditions}

The wps 1 mutants were isolated from an EMS-mutagenized M2 population of Gifu B-129. The wild type L. japonicus (Gifu B-129) and wps 1 mutants were grown in a growth chamber at $22 \pm 2{ }^{\circ} \mathrm{C}$ in $16 \mathrm{~h}$ light and $8 \mathrm{~h}$ dark.

\section{Scanning Electron Microscopy}

Plant materials used for Scanning Electron Microscopy (SEM) were fixed in FAA solution as previously described [17]. And then they were dehydrated by the ethanol solution series, $10 \mathrm{~min}$ for each step and then dried by critical point. Leaves were removed from dried materials as much as necessary. The materials were coated with gold palladium in E-1010 ion sputter. SEM was performed with a Hitachi S-2460 scanning electron microscope (Hitachi, Tokyo) at $15 \mathrm{KV}$. SEM photographs were captured electronically and processed with the Adobe Photoshop 7.0 software (Adobe system, Mountain View, CA).

\section{Histology analysis}

Plants samples were fixed and embedded as previously described [22]. Serial sections were taken at $8 \mu \mathrm{m}$ on a rotary microtome, attached to glass slides with $0.1 \%$ poly-lysine solution, and dried on a warming tray $\left(42{ }^{\circ} \mathrm{C}\right)$. The sections were stained in toluidine blue after removed of the embedded medium and observed under a Carl Zeiss microscope. Images were captured by using a Carl Zeiss digital microscope camera, and then processed with the Adobe Photoshop 7.0 software.

\section{Genetic analysis and mapping}

For genetic analysis, wps 1 mutant was backcrossed with its wild type parent line 'Gifu B-129', and the genetic analysis was conducted in the $\mathrm{B} 2$ population. To generate a mapping population, wps 1 mutant was crossed with the accession Miyakojima MG20 [23]. The mutant plants were identified after screening for the floral phenotype in F2 population. A total of 192 homozygous F2 mutant plants from a F2 population with about 800 individuals, were used in the mapping experiment. To map the wps 1 locus, 36 SSR markers from all six linkage groups were selected from the genetic linkage map of L. japonicus (http://www.kazusa.or.jp/lotus/), and the DNA pools were used for whole genome scanning. Two co-segregated markers, TM0127 and TM0135, were identified and thus the position of wps 1 was determined. The sequence information of the markers used in this experiment is shown in Table 1.

\section{RNA in situ Hybridization}

RNA in situ hybridization with digoxigenin-labeled sense and antisense probes was performed on 8 - $\mu$ m sections of Gifu and wps 1 floral apices, as described by Coen et al. [24]. The transcripts of LjAPI $a$ and LjPIa were generated from cDNA fragment as described before [20], which did not include the region of MADS boxes.

\section{Results}

\section{The wrinkled petals and stamens 1 mutant}

In large scale screening an EMS-mutagenized M2 population of Gifu B-129, a wps 1 mutant was isolated. In the vegetative stage, wps 1 mutant looks similar to the wild type, and the malfunction of wps 1 mutant can be easily
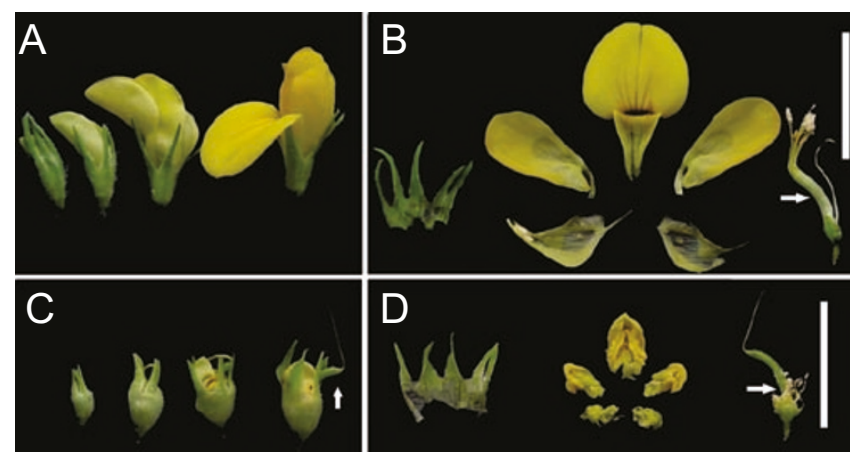

Figure 1 Flowers of wild type and wps 1. (A, B) Wild type flowers. (A) Four floral buds at different developmental stages. (B) Floral organs in a mature flower: five sepals, five petals, and 10 stamens surrounding a carpel (arrow indicates the fused 9 stamens). (C, D) wps 1 flowers. (C) Four floral buds of wps 1 at different developmental stages and in the mature flower, petals and stamens are not expanded and the carpel exposes out (indicated by an arrow); (D) Floral organs in a mature flower of wps 1: the organ numbers are the same as wild type; the development of sepals and carpel of wps 1 is similar to the one of wild type; petals and stamen filaments (indicated by an arrow) are wrinkled or folded, and their sizes are small or shorter than that of wild type. $B a r=1 \mathrm{~cm}$. 


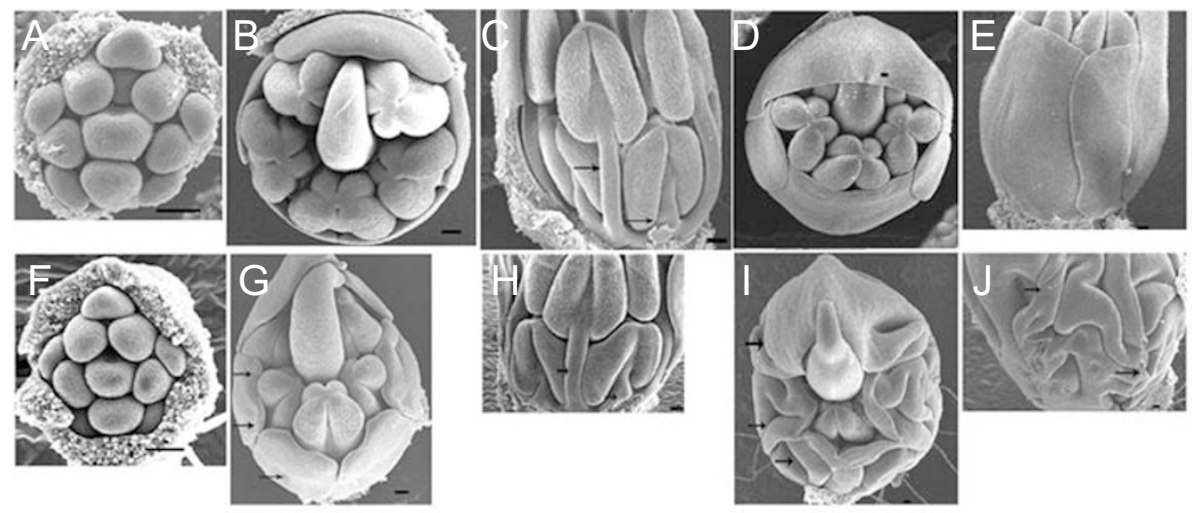

Figure 2 Flower development of wild type and wps1. (A-E) Flower development in wild type. (F-J) the flower development in wps 1 mutants. (A, B, D, F, G, I) Top views. (C, E, H, J) Side views. (A, F) Floral primordia after floral developmental stage 7 as defined in the previous study [20]. (B, C, G, H) Floral primordia at a later stage. $(\mathbf{G}, \mathbf{H})$ Early signs of the malfunction of $w p s 1$ in petals and stamen filaments (indicated by arrows). (D, E, I, J) the floral primordia at the further developed stage. (I, J) The wrinkled petals and stamen filaments are evident (indicated by arrows) in wps 1 . Bar $=50 \mu \mathrm{m}$.

observed in reproductive stages since there is no expanded petal in the mutant flowers and their carpels expose out from floral buds (Figure 1A and 1C). The mutant phenotype can be further revealed by dissecting mature flowers of wps 1 . The petals and stamens of wps 1 mutant are wrinkled or folded in comparison with that of wild type (Figure 1B and 1D); both organs are much shorter than those of wild type in appearance but less so when being stretched (data not shown). However, the development of anthers is not much affected, still bearing fertile pollens. In contrast, both sepal and carpel in wps 1 are relatively normal (Figure 1D). Therefore, wps 1 mutant displays specific defects on the development of petals and stamens.

\section{Genetic analysis of wps 1}

Genetic analysis on wps 1 was conducted by backcrossing the mutant with its parental wild type, Gifu B-129. All plants in B1 displayed normal phenotype as wild type, indicating that the wps 1 mutation is a recessive mutation. In B2 generation, wild type and mutant plants segregated with a 3:1 ratio (167 wild-type plants and 53 mutants). These results show that wps 1 mutant is corresponding to a single nuclear locus. Mapping wps 1 was conducted using a mapping population with about 800 individuals and DNA pools which were randomly selected from 20 mutant plants were used to scanning the whole genome of $L$. japonicus (data not shown). More than 36 SSR markers spreading in different chromosome were tested (Table 1) and then wps 1 was located in the long arm of chromosome 3 by its being co-segregated with a marker TM0127. This has been confirmed by the fine mapping experiment and finally wps 1 was located between two markers, TM0135 and TM0127, at a distance of $2.08 \mathrm{cM}$ and $1.82 \mathrm{cM}$ respectively.

\section{Wps 1 controlling the development of petals and stamens} in late developmental stages

To determine when the function of Wps 1 is required for the normal flower development, we analyzed the flower development at different stages by SEM. The flower development of wps 1 mutants is similar with the one of wild type during early stages, and no malfunction is detected up to floral developmental stage 7 as being defined in the previous study [20], when all floral organ primordia are initiated from the floral primordia and the identities of floral organs suppose to be determined (Figure 2A and 2F). At the late stages of floral organ development, petals and stamens in wps 1 appear abnormal and their shapes become irregular in comparison with wild type (Figure 2B, 2C, 2G and 2H). At subsequently developmental stages, petals and stamens develop further in wild type so as that the petals display smooth abaxial surface and cover the stamens (Figure 2D and 2E); however, the petals and stamens of wps 1 become wrinkled, and do not expand well (Figure 2I and 2J). The arrangement of petals and stamens in wps 1 mutant is irregular, which crush to each other (Figure 2J).

\section{Expression patterns of floral organ identity genes in wps 1 mutants}

The malfunction of wps 1 observed in the petals and stamens raises the question about its relationship with the organ identity genes. In the previous study, a number of organ identity genes in L. japonicus have been isolated [20]. Expression patterns of two representative ones, LjAPIa an A function gene and LjPIa a B function gene, were exam- 

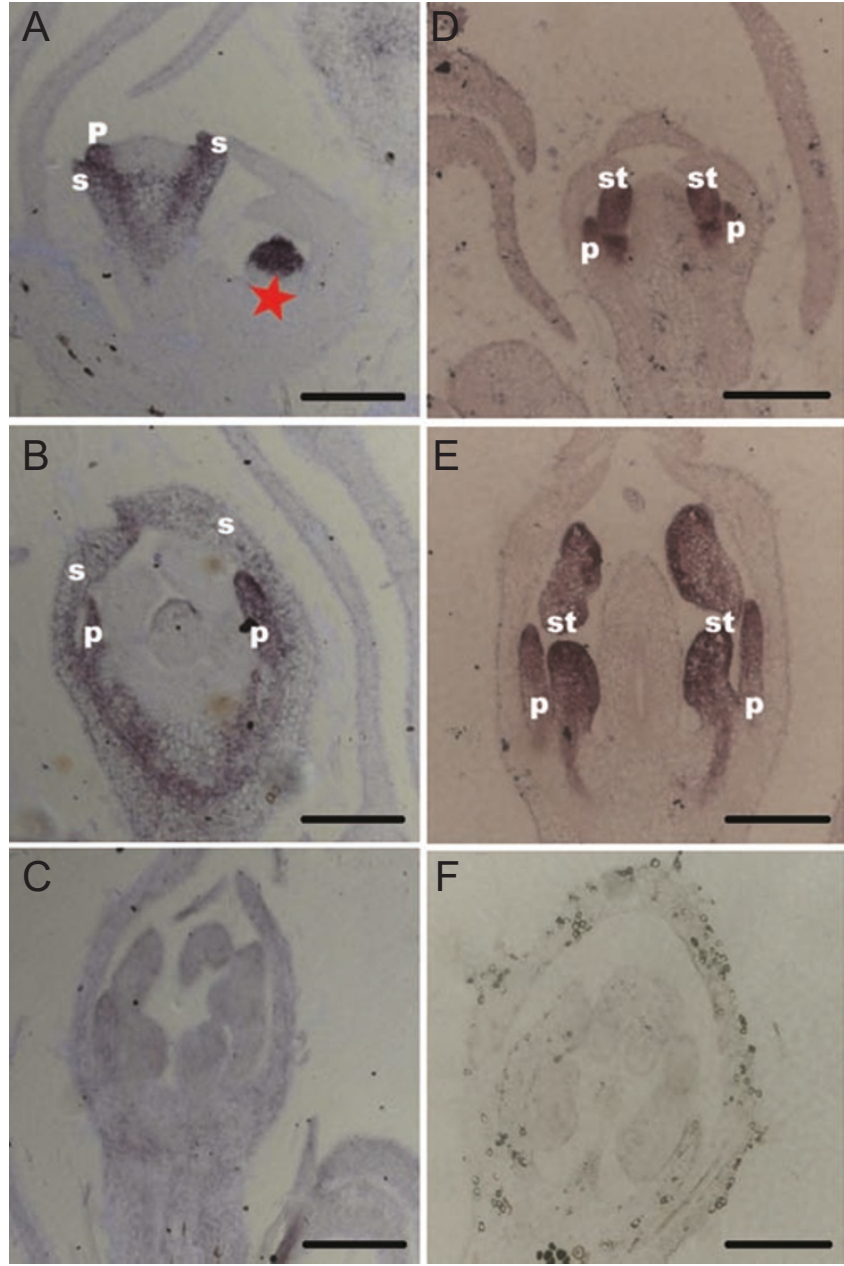

Figure 3 Expression patterns of LjAPIa and LjPIa in wps 1. (A-B) Expression pattern of LjAPla. (A) LjAPla transcripts can be detected in the whole floral primordium at its early development (indicated by a red pentacle), as well as in primordia of sepals and petals. (B) LjAPla is expressed weakly in sepals but strongly in petals at late stage. (C) Control with sense probe of LjAPIa. (D-E) LjPIa is strongly expressed in the primordia of petals and stamens from stage 7 and afterward. (F) Control with sense probe of LjPIa. s, sepal; p, petal; st, stamen. Bar $=100 \mu \mathrm{m}$.

ined in the wps 1 mutant. The LjAPla transcripts were first detected in the whole floral primordium at the early stage, and then were detected at sepals and petals at stage 5 in wps 1 mutants (Figure 3A). At late stages the LjAPla still strongly expressed in petal primordia but weak in sepal primordia (Figure 3B). In a word, the expression pattern of LjAPIa in the wps 1 mutants is similar to those in wild type reported previously [20]. The transcripts of LjPIa were detected in the primordia of petals and stamens in wps 1 mutant flower (Figure 3D and 3E), in the same sites of wild types [20]. These results demonstrate that the expression patterns of floral organ identities genes are the same in wps 1 mutant as in the wild type, suggesting that wps 1 does not affect the expression of organ identity genes.

Subtle morphological alteration of the epidermal cells in petals of wps 1

In the flowers of wps 1, the shapes of all petals are irregular (Figure 1D), but the petal identity is maintained and the floral organ identity genes in wps 1 mutant still possess the same expression pattern as the one in wild type (Figure 3). As a normal papilionoid legume, there are three types of petals in wild type flower of L. japonicus: standard, wing and keel, arranging along a dorsoventral axis of the flower [21], which display different shapes (Figure 1B). Representative cells can be recognized at the epidermal layer among different petals: the conical cells in the standard (Figure 4A); the jigsaw puzzle-shaped cells in

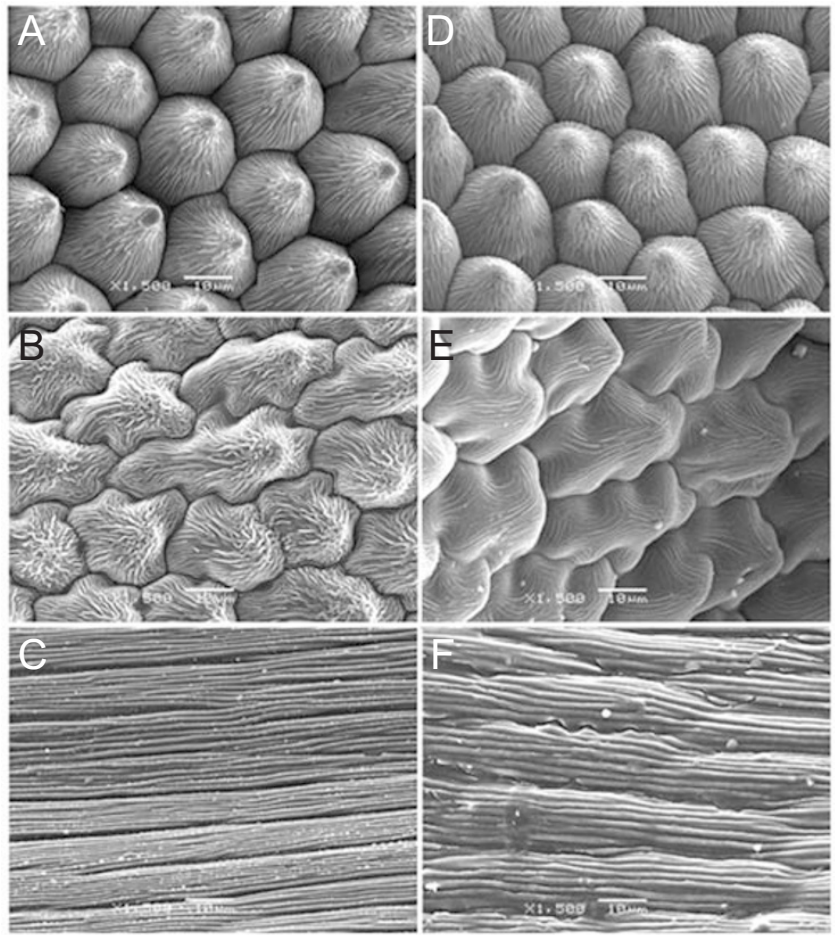

Figure 4 Cellular morphology of adaxial epidermal layer in petals of wild type and wps1. (A-C) Representative cell types in different petals of wild type. (D-F) Representative cell types in different petals of wps 1 mutant. (A, D) Conical cells appear in the standards of both wild type and wps 1 mutant. (B, E) Cells with interlocking jigsaw shape in the wings of both wild type and wps 1 mutant. $(\mathbf{C}, \mathbf{F})$ typical epidermal cells of keels in both wild type and wps 1 mutant share the similar shape, but the width of cells in wps 1 (F) is larger than that of wild type (C). Bar $=10 \mu \mathrm{m}$. 


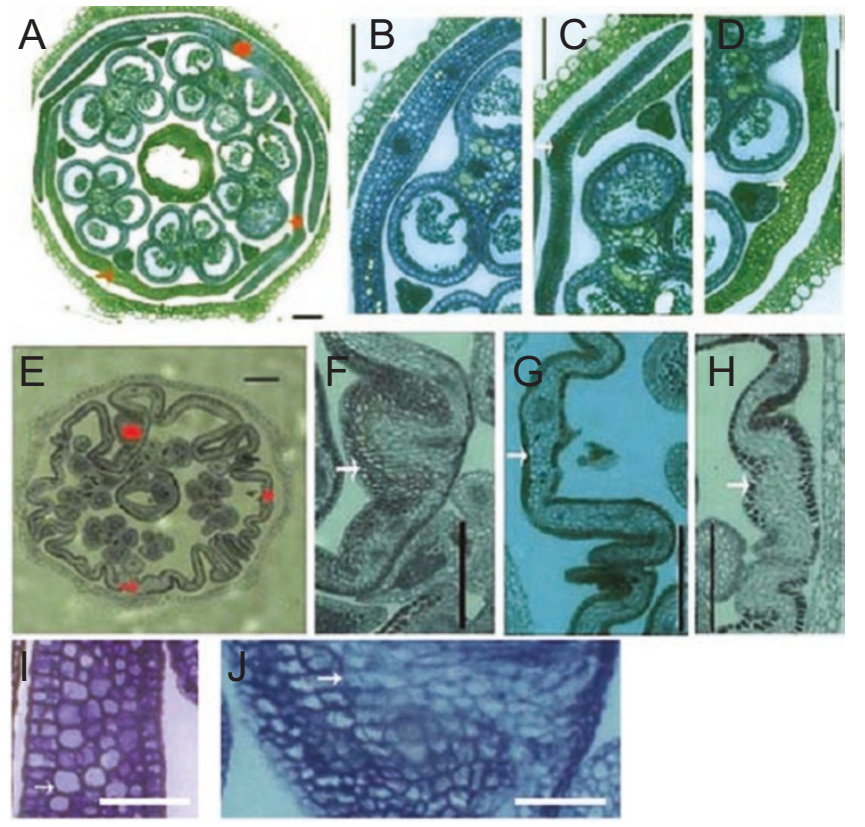

Figure 5 Cellular structure of petals in wild type and wps 1. (A-D, I) Crossing sections of a wild type flower. (E-H, J) Crossing sections of a wps 1 flower. (B-D, I) Close-up of different regions in petals of wild type (from the red marks in A), and 6 to 7 layers of cells in standard (I). (F-H, J) Close-up of knotted regions in petals of wps 1 (from the red marks in $\mathbf{E}$ ), and the malformed and unorganized cells in a knotted region $\mathbf{( J )}$. (A-H) Bar $=100 \mu \mathrm{m} ;(\mathbf{I}, \mathbf{J})$ Bar $=50 \mu \mathrm{m}$.

the wing (Figure 4B); and the wax-covered rectangle cells in the keel (Figure 4C). Recent study showed that these representative cell types could be used as morphological markers to distinguish the dorsoventral identity of different petal types (Our unpublished data). We examined if there was any alteration in the dorsoventral identity among different types of petals by comparing the morphology of the adaxial epidermal cells in mature petals [21] between the wild type and wps 1 mutant. It was found that the characteristic shapes of representative epidermal cells could be clearly identified in the petals of wps 1 , similar to the ones in wild type (Figure $4 \mathrm{~A}-4 \mathrm{~F}$ ). This confirms that there is no detectable alteration at the petal identity in wps 1 . These data also demonstrate that wps 1 has some effects in the cellular development in the L1 or the epidermal layer, since subtle difference could be found in the surfaces of the epidermal cells in the wings (Figure $4 \mathrm{~B}$ and 4E), as well as in the sizes such as the width of cells in the keels of wps 1 is larger than that of wild type (Figure $4 \mathrm{C}$ and $4 \mathrm{~F}$ ).

Histological analysis of petal structure in wild type and wps 1

To investigate the cause of the wrinkled petals and stamen filaments in wps 1 mutant, histological study was conducted. Transversal crossing sections were prepared from mature floral buds of both wild type and wps $1 \mathrm{mu}-$ tant. In comparison with the wild type flower, all petals in wps 1 displayed abnormal curvature due to their irregularly folding (Figure $5 \mathrm{~A}$ and 5E). The arrangement of petals is altered in wps 1 mutant. Petals do not cover each other in the same way as the wild type (Figure 5E). There are knotted structures in the petals, which contain more layers of cells than the normally 6 to 7 layers in wild type (Figure 5 B-5D, 5F-5H). Furthermore, the shape and arrangement of the inner cells in petals of wps 1 mutants are malformed and unorganized in comparison to those of wild type (Figure $4 \mathrm{I}$ and $4 \mathrm{~J})$. All stamen filaments in wps 1 also display the similar abnormal phenotype (data not shown). These data suggest that the development of petals and stamen filaments in wps 1 mutant is somehow affected at the cellular level, and processes in both cell division and elongation could be malfunctioned.

\section{Discussion}

Wps 1 could be a downstream component in the B function during flower development in L. japonicus

In the past twenty years, the ABC model of flower development has been widely promulgated. This model, based on genetic experiments in Antirrhinum and Arabidopsis $[1,2]$, is striking in its simplicity and applicable in a wide range of angiosperm species. However, the development of floral organs requires not only the $\mathrm{ABC}$ function genes but also the downstream factors to elaborate their final shape. Although many putative targets of the floral organ identity genes were identified using micro-arrays analysis [6,27], robust analysis on their function is few and so far only NAP has been shown to be a direct target of AP3/PI [5].

In this study, we analyzed a novel floral mutant wps 1 . In our mutagenesis experiment, more than five mutants have been identified, which displayed the similar phenotype as wps 1, and our preliminary data indicate they are allelic to $w p s 1$, suggesting $W p s 1$ could be a hot spot susceptible to the mutagenesis (unpublished data). It is found that the malfunction of wps 1 is mainly observed in petals and stamens, suggesting the activity of $W p s 1$ should be within the functional domain of the B function genes. It was found that two of the floral organ identity genes displayed the same expression patterns in wps 1 as in wild type, indicating there is no alteration of organ identities during the flower development in the mutant. Furthermore, the dorsoventral identity seems to be well maintained as the characteristic shapes of representative epidermal cell types in different petals were found in both wild type and wps 1 .

The flower development of $L$. japonicus can be divided 
into two phases: phase I when the floral organ identities are determined, and phase II when the elaboration process to form fine structures within floral organ takes place. Up to 7 floral developmental stages in L. japonicus have been defined in our previous study [20]. Stage 7 should be considered as the end of phase I, when floral organ identities are determined (Figure 2A, [20]). In wps 1 mutant, malfunction in flower development commences after stage 7 (Figure $2 \mathrm{~B}$ and $2 \mathrm{G}$ ), confirming the function of $W p s 1$ should take place after the determination of floral organ identities. Detailed analysis and further definition of late floral organ development are needed to address the question about precise time when $W p s 1$ take part in the development of floral organs in L. japonicus. However, taken the specific effect of the wps 1 mutant on the late development of petal and stamen, Wps 1 is most likely a component of B function during flower development in L. japonicus.

Function of Wps1 during flower development of L. japonicus

MADS-box transcription factors are key regulators during floral organ morphogenesis $[4,26]$, and their role to determine organ identity could be through targeting other downstream components. This raises the question about the function of the downstream components during floral organ development. In this study, we analyze a novel locus Wps 1 in L. japonicus, which could be such a downstream component and should play an important role in the control of development of both petals and stamens mainly in the late floral developmental stages.

The main defect of wps 1 was found in the petals and stamen filaments, which are wrinkled or irregularly folded (Figure $1 \mathrm{D}$ ). The histological investigation indicated that the malformed and unorganized cellular development in these two floral organs could account for the mutant phenotype in wpsl. It is shown that in wpsl, petals and stamens are irregularly folded (Figure 5E), and knotted structures with more cell layers appear in petals (Figure 5 F-5H). Moreover, the inner cells of petals and stamens in the mutant are malformed and unorganized (Figure $5 \mathrm{~J}$ ). These data strongly indicate that the cell division and cell elongation in the petals and stamens of the mutant may be affected, suggesting a role of Wps 1 in the control of cellular development during the late floral organ development.

Cell division and elongation processes are generally important for the development of plants, and after floral organ identities are determined, there might be different mechanisms, under which controls, lateral organs acquire different rate of cell division and specific expansion pattern along different axes during late floral development stages [28]. It has been shown that maintaining the pattern of cell division is crucial for the development of a flat leaf surface.
In cin mutant of Antirrhinum, the leaf margin is rumpled as a result of the slower arrest of cell divisions at the leaf margins relative to the middle of the leaf, and a comparable phenotype is seen in jaw-D mutation of Arabidopsis [29, 30]. In both cases, TCP-domain genes [31] and regulation of their expressions are responsible for the development of flat and curved leaf surface. In Arabidopsis, NAP, a direct target of B function genes has been identified, which is regulated by the Arabidopsis class B protein APETALA3 and is thought to take part in the process in the transition between growth from cell division to cell elongation in petals and stamens [5]. In the late floral development, floral organs commence growth in a fast rate, and it is expected that a regulatory mechanism should monitor the cell division and elongation so as to determine the final morphology of floral organs. With the cloning of Wps 1 in the near future, it would be possible to make the comparison of the functions and activities of $W p s 1$ and $N A P$, and address the question if there is a conserved mechanism in the control of floral organ morphogenesis during late development among different species.

\section{Conclusion}

L. japonicus, a legume model plant, provides an amenable system to screen the mutants and dissect the pathway in the control of floral organ development. We have been exploiting L. japonicus, and using the EMS mutagenesis approach to isolate novel floral mutations. In this study, a novel mutation, wps 1 was analyzed, which corresponds to the malfunction of $W p s 1$, a potential downstream component of $\mathrm{B}$ function organ identity genes. Wps 1 could play a key role in the control of cellular differentiation during the late flower development stages. Further study and cloning of Wps 1 in L. japonicus will be helpful to analyze the link between the early and the late developmental control during floral morphogenesis, as well as the function and regulation of downstream components in the B function pathway.

\section{Acknowledgements}

The authors thank Mr Qin Peng Mong, Xiao Feng Li, Satoshi Tabata, Shusei Sato and Jun Yang for their help to conduct the experiment, and Dr Liang Huang for helpful revision of the manuscript. This research was financially supported by the National Natural Science Foundation of China (Grant No. 30430330).

\section{References}

1 Coen ES, Meyerowitz EM. The war of the whorls: genetic interactions controlling flower development. Nature 1991; 353:31-37. 
2 Ma H, dePamphilis C. The ABCs of floral evolution. Cell 2000; 101:5-8.

3 Colombo 1, Franken J, Koetje E, et al. The petunia MADS box gene FBP11 determines ovule identity. Plant Cell 1995; 7:18591868.

4 Pelaz S, Ditta GS, Baumann E, Wisman E, Yanofsky M. B and C floral organ identity functions require SEPALLATA MADS-box genes. Nature 2000; 405: 200-203.

5 Sablowski RW, Meyerowitz EM. A homolog of NO APICAL $M E R I S T E M$ is an immediate target of the floral homeotic genes APETALA3/PISTILLATA. Cell 1998; 92:93-103.

6 Zik M, Irish VF. Global identification of target genes regulated by APETALA3 and PISTILLATA floral homeotic gene action. Plant Cell 2003; 15:207-222.

7 Wellmer F, Riechmann JL, Alves-Ferreira M, Meyerowitz EM. Genome-wide analysis of spatial gene expression in Arabidopsis flowers. Plant Cell 2004; 16:1314-1326.

8 Handberg K, Stougaard J. Lotus japonicus, an autogamous diploid legume species for classical and molecular genetics. Plant J 1992; 2:487-496.

9 Perry JA, Wang TL, Welham TJ, et al. A TILLING reverse genetics tool and a web-accessible collection of mutants of the legume Lotus japonicus. Plant Physiol 2003; 131:866-871.

10 Somers DA, Samac DA, Olhoft PM. Recent advances in legume transformation. Plant Physiol 2003; 131:892-899.

11 VandenBosch KA, Stacey G. Summaries of legume genomics projects from around the globe: community resources for crops and models. Plant Physiol 2003; 131: 840-865.

12 Sato S, Kaneko T, Nakamura Y, et al. Structural analysis of the Lotus japonicus genome. I. Sequence features and mapping of fifty-six TAC clones which cover the $5.4 \mathrm{Mb}$ regions of the genome. DNA Res 2001; 8:311-318.

13 Imaizumi-Anraku H, Kawaguchi M, Koiwa H, Akao S, Syono K. Two ineffective nodulating mutants of Lotus japonicus: different phenotypes caused by the blockage of endocytotic bacterial release and nodule maturation. Plant Cell Physiol 1997; 38:871881.

14 Schauser L, Handberg K, Sandal N, et al. Symbiotic plant mutants deficient in nodule establishment identified after TDNA transformation of Lotus japonicus. Mol Gen Genet 1998; 259:414-423.

15 Hayashi M, Miyahara A, Sato S, et al. Construction of a genetic linkage map of the model legume Lotus japonicus using an intraspecific F2 population. DNA Res 2001; 8:301-310.

16 Sandal N, Krusell L, Radutoiu S, et al. A genetic linkage map of the model legume Lotus japonicus and strategies for fast mapping of new loci. Genetics 2002; 161: 1673-1683.
17 Ferrandiz C, Navarro C, Gomez MD, Canas LA, Beltran JP. Flower development in Pisum sativum: from the war of the whorls to the battle of the common primordium. Dev Genet 1999; 25:280-290.

18 Benlloch R, Navarro C, Beltran JP, Canas LA. Floral development of the model legume Medicago truncatula: ontogeny studies as a tool to better characterize homeotic mutations. Sex Plant Reprod 2003; 15:231-241.

19 Tucker SC. Overlapping organ initiation and common primordia in flowers of Pisum sativum (Leguminosae: Papilionoideae). Am J Bot 1989; 74:714-729.

20 Dong ZC, Zhao Z, Liu CW, et al. Floral patterning in Lotus japonicus. Plant Physiol 2005; 137:1271-1282.

21 Feng XZ, Zhao Z, Tian ZX, et al. Control of petal shape and floral zygomorphy in Lotus japonicus. Proc Natl Acad Sci U S A 2006 Mar; in press (online: www.pnas.org/cgi/doi/10.1073/ pnas.0600681103)

22 Jackson D, Veit B, Hake S. Expression of maize KNOTTED1 related homeobox genes in the shoot apical meristem predicts patterns of morphogenesis in the vegetative shoot. Development 1994; 120:405-413.

23 Kawaguchi M, Motomura T, Imaizumi-Anraku H, Akao S, Kawasaki S. Providing the basis for genomics in Lotus japonicus: the accession Miyakojima and Gifu are appropriate crossing partners for genetic analyses. Mol Genet Genomics 2001; 266:157-166.

24 Coen ES, Romero JM, Doyle S, et al. floricaula: a homeotic gene required for flower development in Antirrhinum majus. Cell 1990; 63:1311-1322.

25 Choi HK, Mun JH, Kim DJ, et al. Estimating genome conservation between crop and model legume species. Proc Natl Acad Sci USA 2004; 101:15289-15294.

$26 \mathrm{Ng}$ M, Yanofsky MF. Function and evolution of the plant MADSbox gene family. Nat Rev Genet 2001; 2:186-195.

27 Bey M, Stuber K, Fellenberg K, et al. Characterization of Antirrhinum petal development and identification of target genes of class B MADS box gene DEFICIENS. Plant Cell 2004; 16:31973215 .

28 Donnelly PM, Bonetta D, Tsukaya H, et al. Cell cycling and cell enlargement in developing leaves of Arabidopsis. Dev Biol 1999; 215:407-419.

29 Nath U, Crawford BC, Carpenter R, Coen E. Genetic control of surface curvature. Science 2003; 299:1404-1407.

30 Palatnik JF, Allen E, Wu X, et al. Control of leaf morphogenesis by microRNAs. Nature 2003; 425:257-263.

31 Cubas P, Lauter N, Doebley J, Coen E. The TCP domain: a motif found in proteins regulating plant growth and development. Plant J. 1999; 18:215-222. 